\section{Genzyme erhält für die Zulassung von Mozobil in Europa die «Positive Opinion»}

Der Ausschuss für Humanarzneimittel (CHMP) der Europäischen Arzneimittelbehörde hat die so genannte «Positive Opinion» für eine Zulassung von Mozobil ${ }^{\circledR}$ (Plerixafor zur Injektion) erteilt. In der EU wird Mozobil ${ }^{\circledR}$ in Kombination mit dem Wachstumsfaktor G-CSF (granulocyte colony-stimulation factor) voraussichtlich zugelassen, um die Mobilisierung von hämatopoetischen Stammzellen in das periphere Blutsystem zu verstärken. Dadurch sollen Stammzellen für die autologe Transplantation bei Patienten mit Lymphomen und Multiplem Myelom gewonnen werden, deren Zellen nicht in ausreichendem Maße mobilisiert werden können.

«Für viele Patienten mit Lymphomen oder
Multiplem Myelom ist dieser entscheidende Schritt zur Transplantation die einzige Hoffnung auf eine Remission oder Heilung», sagte Gerhard Ehninger, Professor für Innere Medizin an der Technischen Universität Dresden und Vorsitzender der Deutschen Gesellschaft für Hämatologie und Onkologie. «Mozobil ${ }^{\circledR}$ stellt eine innovative Therapie dar, die nach ihrer Zulassung diesen Patienten die Chance auf eine rechtzeitige und erfolgreiche Transplantation bieten kann.»

Für eine Transplantation von Blut-Stammzellen muss eine Mindestzahl solcher Zellen gewonnen werden. Bei vielen Patienten muss dieser Vorgang mehrere Tage lang über 3-4 Stunden wiederholt werden. Aber auch dann gelingt es in manchen Fällen nicht, genügend Zellen zu mobilisieren, und eine Transplantation ist dann nicht möglich. Klinische Studien belegen, dass Mozobil ${ }^{\circledR}$ bei Patienten mit
Lymphomen oder Multiplem Myelom rasch und wirksam einen Anstieg der im Blut zirkulierenden Stammzellen bewirken kann.

In Europa haben Patienten im Rahmen des Compassionate-Use-Programms und in so genannten Named-Patient-Programmen Mozobil ${ }^{\circledR}$ erhalten.

Genzyme hat im Dezember 2008 die FDAZulassung für Mozobil ${ }^{\circledR}$ erhalten. Das Unternehmen plant, in bis zu 60 weiteren Ländern die Zulassung zu beantragen.

Weitere Informationen bei

Genzyme GmbH

Bianca Zeiner

PR Specialist

Tel. +49 6102 3674-349

bianca.zeiner@genzyme.com
Verbesserte Therapieoption für Hämophilie-APatienten:

\section{ReFacto AF $^{\circledR}$ seit Juni 2009 verfügbar}

Seit dem 1. Juni 2009 steht Hämophilie-APatienten mit ReFacto $\mathrm{AF}^{\circledR}$ (Moroctocog alfa) in der Europäischen Union eine verbesserte Therapieoption zur Verfügung. ReFacto $\mathrm{AF}^{\circledR}$ ist das einzig verfügbare Faktor-VIIIKonzentrat, das von der Zellkultur bis zum fertigen Präparat ohne den Einsatz von exogenen humanen oder tierischen Eiweißen auskommt. Dadurch besitzt ReFacto $\mathrm{AF}^{\circledR}$ die derzeit höchste Virussicherheit, bezogen auf neue, bislang unbekannte Krankheitserreger. Die Wirksamkeit und Sicherheit von Moroctocog alfa zur Behandlung und Prophylaxe von Blutungsepisoden wurde in 2 klinischen Studien erneut belegt.

Moroctocog alfa wird aus Ovarialzelllinien des chinesischen Hamsters (CHO-Zelllinien) sezerniert. Mit dem neuen, verbesserten Herstellungsprozess ist bereits das Medium der CHOZelllinie frei von humanem Serumalbumin und anderen Proteinen humanen oder tierischen Ursprungs. Als zusätzlicher Sicherheitsschritt wurde eine Nanofiltration eingefügt. Dadurch werden Viren sowie weitere mögliche Rückstände zuverlässig entfernt. Auch diese Nanofiltration wird in der Gruppe der rekombinanten, in Deutschland erhältlichen Faktor VIII-Präparate nur bei ReFacto $\mathrm{AF}^{\circledR}$ verwendet.

Die Weiterentwicklung des seit 1999 zugelassenen Produktes ReFacto ${ }^{\circledR}$ mit dem gleichen Wirkstoff Moroctocog alfa verfügt über eine gute hämostatische Wirksamkeit und hohe Sicherheit in Bezug auf die Entwicklung von Hemmkörpern, wie im Rahmen von 2 Studien [1] erneut belegt wurde.

Den Patienten steht mit ReFacto $\mathrm{AF}^{\circledR}$ ein
Faktor-VIII-Präparat zur Verfügung, das noch reiner ist als ReFacto ${ }^{\circledR}$ und - bezogen auf neue, bislang unbekannte Krankheitserreger die derzeit höchste Virussicherheit aufweist.

\section{Literatur}

1 Recht $\mathrm{M}$ et al. Clinical evaluation of moroctocog alfa (AF-CC), a new generation of B-domain deleted recombinant factor VIII (BDDrFVIII) for treatment of haemophilia A: demonstration of safety, efficacy, and pharmacokinetic equivalence to full-length recombinant factor VIII. Haemophilia (2009), DOI: 10.1111/j.13652516.2009.02027x.

Weitere Informationen bei

SanCom CCS GmbH

Kathrin Schuster

Tel. +49 6123 70558-35, Fax -69

schuster@sancom-ccs.de

\title{
Ticker+++ Ticker+++ Ticker+++ Ticker+++ Ticker+++ Ticker+++ Ticker+++
}

Bayer Vital GmbH. Rivaroxaban (Xarelto $\left.{ }^{\circledR}\right)$, der erste direkte, orale Faktor-Xa-Inhibitor, ist seit Zulassung im September 2008 für die Prävention venöser Thromboembolien (VTE) bei erwachsenen Patienten nach elektiven Knie- und Hüftgelenkersatzoperationen erfolgreich im Einsatz. Ab sofort steht das Präparat auch als 5er Packung (N1) zur Verfügung - zusätzlich zur N1-10-Packung mit 10 Tabletten sowie der 30 Tabletten enthaltenden N2Einheit.

Bayer Vital GmbH

Dr. Herbert Schäfer

Tel +49 214 3051-109, Fax -517

herbert.schaefer@bayerhealthcare.com
Vifor Deutschland GmbH. Mit Ferinject ${ }^{\circledR}$ steht eine parenterale Eisentherapie für Patienten zur Verfügung. Aufgrund des dextranfreien und hochstabilen Zucker-Eisen-Komplexes kann ohne vorherige Testdosis eine Infusion von bis zu 1000 mg über mindestens 15 Minuten (maximal $1000 \mathrm{mg} /$ Woche) infundiert oder $200 \mathrm{mg}$ per Bolusinjektion pro Tag appliziert (maximal $3 \times 200 \mathrm{mg} /$ Woche) werden.

MW Office PR

Dr. Stefanie Wesche

Tel. +49 89 96086-368, Fax -333

stefanie.wesche@mwoffice.de
Essex Pharma GmbH. Im operativen intensivmedizinischen Bereich nehmen Pilzinfektionen zu. Zur Prophylaxe nach Organtransplantation, aber auch für die Deeskalationtherapie nach i. v.-Behandlung von systemischen Pilzinfektionen bietet das oral verfügbare Posaconazol $\left(\mathrm{NOXAFIL}^{\circledR}\right)$ eine Therapieoption mit breitem Wirkspektrum. Eine Studie mit einer i.v.-Formulierung von Posaconazol läuft bereits.

Publicis Health

Frau Saskia Nießner

Tel. +49 221 912719-564, Fax -764 saskia.niessner@ publicis-health.de 